\title{
When Is the Complement of the Comaximal Graph of a Commutative Ring Planar?
}

\author{
S. Visweswaran and Jaydeep Parejiya \\ Department of Mathematics, Saurashtra University, Rajkot 360 005, India \\ Correspondence should be addressed to S. Visweswaran; s_visweswaran2006@yahoo.co.in
}

Received 10 February 2014; Accepted 6 March 2014; Published 6 April 2014

Academic Editors: D. F. Anderson, B. Bakalov, and A. Zimmermann

Copyright (C) 2014 S. Visweswaran and J. Parejiya. This is an open access article distributed under the Creative Commons Attribution License, which permits unrestricted use, distribution, and reproduction in any medium, provided the original work is properly cited.

\begin{abstract}
Let $R$ be a commutative ring with identity. In this paper we classify rings $R$ such that the complement of comaximal graph of $R$ is planar. We also consider the subgraph of the complement of comaximal graph of $R$ induced on the set $S$ of all nonunits of $R$ with the property that each element of $S$ is not in the Jacobson radical of $R$ and classify rings $R$ such that this subgraph is planar.
\end{abstract}

\section{Introduction}

All rings considered in this paper are commutative with identity $1 \neq 0$. The graphs considered here are undirected and simple. Sharma and Bhatwadekar in [1] introduced a graph $\Omega(R)$ on a commutative ring $R$, whose vertices are the elements of $R$ and two distinct vertices $x$ and $y$ are adjacent if and only if $R x+R y=R$. In [1, Theorem 2.3], the authors showed that $\chi(\Omega(R))<\infty$ if and only if the ring $R$ is finite and in this case $\chi(\Omega(R))=\omega(\Omega(R))=t+l$, where $t$ and $l$ denote, respectively, the number of maximal ideals of $R$ and number of units of $R$. In [2], Maimani et al. named the graph studied by Sharma and Bhatwadekar as the comaximal graph $\Gamma(R)$ of $R$. Also in [2], the authors studied the subgraphs $\Gamma_{1}(R), \Gamma_{2}(R)$, and $\Gamma_{2}(R) \backslash J(R)$, where $\Gamma_{1}(R)$ is the subgraph of $\Gamma(R)$ induced on the set of units of $R, \Gamma_{2}(R)$ is the subgraph of $\Gamma(R)$ induced on the set of nonunits of $R$, and $\Gamma_{2}(R) \backslash J(R)$ is the subgraph of $\Gamma(R)$ induced on the set of nonunits of $R$ which are not in $J(R)$ $=$ the Jacobson radical of $R$. Several other researchers also investigated the comaximal graphs of rings [3-5]. In [6], Gaur and Sharma studied the graph whose vertices are the elements of a ring $R$ and two distinct vertices $x$ and $y$ are adjacent if and only if there exists a maximal ideal of $R$ containing both $x$ and $y$. They called this graph the maximal graph of the ring $R$.

Let $G=(V, E)$ be a simple graph. Recall from [7, Definition 1.1.13] that the complement $G^{c}$ of $G$ is defined by taking $V\left(G^{c}\right)=V(G)=V$ and two distinct vertices $u$ and $v$ are adjacent in $G^{c}$ if and only if they are not adjacent in G. Thus the maximal graph of a ring studied in [6] is the complement of the comaximal graph of a ring studied by Sharma and Bhatwadekar in [1].

For any set $A,|A|$ denotes the cardinality of $A$. For any ring $R, U(R)$ and $N U(R)$ denote, respectively, the set of units of $R$ and the set of nonunits of $R$. We denote $R \backslash\{0\}$ by $R^{*}$ and the set of all maximal ideals of $R$ by $\operatorname{Max}(R)$. For the sake of convenience, for any ring $R$ with at least two maximal ideals we denote $\Gamma_{2}(R) \backslash J(R)$ by $G(R)$. For any $n \geq 2, \mathbf{Z}_{n}$ denotes the ring of integers modulo $n$. For any prime number $p$ and $n \geq 1, \mathbf{F}_{p^{n}}$ denotes the finite field with exactly $p^{n}$ elements.

We first recall the following definitions and results from graph theory. A graph $G=(V, E)$ is said to be complete if every pair of distinct vertices of $G$ are adjacent in $G$. A complete graph on $n$ vertices is denoted by $K_{n}$ [7, Definition 1.1.11]. A graph $G=(V, E)$ is said to be bipartite if the vertex set can be partitioned into two nonempty subsets $X$ and $Y$ such that each edge of $G$ has one end in $X$ and another one in $Y$. The pair $(X, Y)$ is called a bipartition of $G$. A bipartite graph $G$ with bipartition $(X, Y)$ is denoted by $G(X, Y)$. A bipartite graph $G(X, Y)$ is said to be complete if each vertex of $X$ is adjacent to all the vertices of $Y$. If $G(X, Y)$ is a complete bipartite graph with $|X|=m$ and $|Y|=n$, then it is denoted by $K_{m, n}$ [7, Definition 1.1.12]. Let $G=(V, E)$ be a graph. By a clique of $G$, we mean a complete subgraph of $G[7$, Definition 1.2.2]. We say that the clique number of $G$ equals $n$ if $n$ is 
the largest positive integer such that $K_{n}$ is a subgraph of $G$ [7, page 185]. The clique number of a graph $G$ is denoted by the notation $\omega(G)$. If $G$ contains $K_{n}$ as a subgraph for all $n \geq 1$, then we set $\omega(G)=\infty$.

A graph $G$ is said to be planar if it can be drawn in a plane in such a way that no two edges of $G$ intersect in a point other than a vertex of $G$ [7, Definition 8.1.1]. Two adjacent edges of a graph $G$ are said to be in series if their common vertex is of degree two [8, page 9]. Two graphs are said to be homeomorphic if one graph can be obtained from the other graph by the creation of edges in series (i.e., by insertion of vertices of degree two) or by the merger of edges in series $[8$, page 100]. Recall from [8, page 93] that $K_{5}$ is referred to as Kuratowski's first graph and $K_{3,3}$ is referred to as Kuratowski's second graph. A celebrated theorem of Kuratowski says that a necessary and sufficient condition for a graph $G$ to be planar is that $G$ does not contain either of Kuratowski's two graphs or any graph homeomorphic to either of them $[8$, Theorem 5.9].

Motivated by the interesting theorems proved on comaximal graphs of rings in [1-5] and also the theorems proved on its complement in [6], in this paper we attempt to characterize commutative rings $R$ such that $(\Omega(R))^{c}$ is planar, where $\Omega(R)$ is the comaximal graph of a ring $R$. Since any subgraph of a planar graph is planar, it follows that $(G(R))^{c}\left(=\left(\Gamma_{2}(R) \backslash\right.\right.$ $\left.J(R))^{c}\right)$ is planar if $(\Omega(R))^{c}$ is planar. Hence we first focus our study on investigating when $(G(R))^{c}$ is planar and at the end of this paper we classify the rings $R$ for which $(\Omega(R))^{c}$ is planar. While investigating when $(G(R))^{c}$ is planar, we assume that $R$ has at least two maximal ideals.

In view of Kuratowskis Theorem, [8, Theorem 5.9] we introduce the following definitions.

Let $G=(V, E)$ be a graph.

(i) We say that $G$ satisfies $K u_{1}$ if $G$ does not contain $K_{5}$ as a subgraph.

(ii) We say that $G$ satisfies $K u_{2}$ if $G$ does not contain $K_{3,3}$ as a subgraph.

(iii) We say that $G$ satisfies $K u_{1}^{*}$ if $G$ satisfies $K u_{1}$ and, moreover, $G$ does not contain any subgraph homeomorphic to $K_{5}$.

(iv) We say that $G$ satisfies $K u_{2}^{*}$ if $G$ satisfies $K u_{2}$ and, moreover, $G$ does not contain any subgraph homeomorphic to $K_{3,3}$.

If a graph $G$ is planar, then it follows from Kuratowski's theorem [8, Theorem 5.9] that $G$ satisfies both $K u_{1}^{*}$ and $K u_{2}^{*}$. Hence $G$ satisfies both $K u_{1}$ and $K u_{2}$. It is interesting to note that a graph $G$ may be nonplanar even if it satisfies both $K u_{1}$ and $\mathrm{Ku}_{2}$. For example of this type refer to [8, Figure 5.9(a), page 101] and the graph $G$ in this example does not satisfy $K u_{2}^{*}$. It is not difficult to construct an example of a graph $G$ such that $G$ satisfies $K u_{1}$ but $G$ does not satisfy $K u_{1}^{*}$.

Let $R$ be a ring with at least two maximal ideals. We now give a brief summary of the results proved in this paper. It is shown in Lemma 8 that if $(G(R))^{c}$ satisfies $K u_{1}$ or $K u_{2}$, then $|\operatorname{Max}(R)| \leq 3$. For a ring $R$ with exactly two maximal ideals, it is proved in Theorem 19 that $(G(R))^{c}$ satisfies $K u_{1}$ if and only if $(G(R))^{c}$ satisfies $K u_{2}$ if and only if $(G(R))^{c}$ is planar. Moreover, the statement (2) of Theorem 19 describes such rings up to isomorphism. If $R$ is a ring with exactly three maximal ideals, then it is shown in Theorem 24 that $(G(R))^{c}$ satisfies $K u_{1}$ if and only if $(G(R))^{c}$ satisfies $K u_{2}$ if and only if $(G(R))^{c}$ is planar if and only if $R \approx \mathbf{Z}_{2} \times \mathbf{Z}_{2} \times \mathbf{Z}_{2}$ as rings.

Let $R$ be a ring with at least two maximal ideals. As $(G(R))^{c}$ is a subgraph of $(\Omega(R))^{c}$, we obtain from Lemma 8 that if $(\Omega(R))^{c}$ satisfies $K u_{1}$ or $K u_{2}$, then $|\operatorname{Max}(R)| \leq 3$. It is proved in Theorem 32 that, for a ring $R$ with exactly two maximal ideals, $(\Omega(R))^{c}$ satisfies $K u_{1}$ and $K u_{2}$ if and only if $(\Omega(R))^{c}$ satisfies $K u_{1}$ if and only if $(\Omega(R))^{c}$ is planar. Also, in statement (3) of Theorem 32, rings $R$ with $|\operatorname{Max}(R)|=2$ such that $(\Omega(R))^{c}$ is planar are listed up to isomorphism of rings. In Remark 34, an example of a ring $R$ with $|\operatorname{Max}(R)|=2$ is provided to illustrate that $(\Omega(R))^{c}$ which satisfies $K u_{2}$ need not imply that it satisfies $K u_{1}$. Moreover, in Theorem 35, rings $R$ with $|\operatorname{Max}(R)|=2$ such that $(\Omega(R))^{c}$ satisfies $K u_{2}$ are listed up to isomorphism of rings. For a ring $R$ with $|\operatorname{Max}(R)|=3$, it is proved in Theorem 36 that $(\Omega(R))^{c}$ satisfies $K u_{1}$ if only if $(\Omega(R))^{c}$ satisfies $K u_{2}$ if and only if $(\Omega(R))^{c}$ is planar if and only if $R \approx \mathbf{Z}_{2} \times \mathbf{Z}_{2} \times \mathbf{Z}_{2}$ as rings.

In Section 6, quasilocal rings $(R, M)$ which are not fields are considered and necessary and sufficient conditions are determined for $(\Omega(R))^{c}$ to be planar. It is proved in Theorem 37 that, for a quasilocal ring $(R, M)$ which is not a field, $(\Omega(R))^{c}$ satisfies $K u_{1}$ and $K u_{2}$ if and only if $(\Omega(R))^{c}$ satisfies $K u_{1}$ if and only if $|M| \leq 4,|R / M| \leq 4$, and $|R| \leq 16$ if and only if $(\Omega(R))^{c}$ is planar. It is observed in Lemma 38 that for a quasilocal ring $(R, M)$ which is not a field, $(\Omega(R))^{c}$ satisfies $K u_{2}$ if and only if $|M| \leq 5,|R / M| \leq 5$, and $|R| \leq 25$. In Remark 40, up to isomorphism of rings, all local rings which are not fields of order 4,8,9,25 and all local rings $(R, M)$ with $|R|=16$ and $|M|=4$ are mentioned with the help of the theorems proved in [9] and from the results proved in [10].

\section{Preliminaries}

As is mentioned in the introduction, the rings considered in this paper are commutative with identity $1 \neq 0$. In this section we state and prove several lemmas which are needed for proving the main results of this paper.

Lemma 1. Let $R$ be a ring which admits at least one nonzero nonunit. If the set of nonunits of $R$ is finite, then the ring $R$ is finite.

Proof. Suppose that $R$ has an infinite number of units. Let " $a$ " be any nonzero nonunit of $R$. Then $\{u a \mid u \in U(R)\} \subseteq$ $N U(R)=$ the set of all nonunits of $R$. As $N U(R)$ is finite, there exist $1 \leq i_{1}<i_{2}<i_{3}<\cdots$ such that $u_{i_{1}} a=u_{i_{j}}$ a for $j=2,3, \ldots$. So $\left(u_{i_{1}}-u_{i_{j}}\right) a=0$ for $j=2,3, \ldots$ which implies that $u_{i_{1}}-u_{i_{j}}$ are distinct nonunits of $R$, for $j=2,3, \ldots$ Hence we obtain that $N U(R)$ is not finite. This is a contradiction. Thus $U(R)$ must be finite. Therefore, $R$ is a finite ring.

Lemma 2. Let $n \geq 4$. If the ring $R$ is such that $\omega\left((G(R))^{c}\right) \leq n$, then $|\operatorname{Max}(R)| \leq n-1$. 
Proof. Suppose that $R$ has more than $n-1$ maximal ideals. Let $\left\{M_{1}, M_{2}, M_{3}, \ldots, M_{n}\right\} \subseteq \operatorname{Max}(R)$. Let $a_{i} \in$ $\left(\bigcap_{j \in\{1,2, \ldots, n\} \backslash\{i\}} M_{j}\right) \backslash M_{i}$ for $i=1,2, \ldots, n$ and $a_{n+1} \epsilon$ $\left(M_{n-1} \cap M_{n}\right) \backslash\left(\bigcup_{j \in\{1,2, \ldots, n-2\}} M_{j}\right)$. We claim that the subgraph of $(G(R))^{c}$ induced on $\left\{a_{i} \mid i \in\{1,2, \ldots, n+1\}\right\}$ is a clique. Let $i, j \in\{1,2, \ldots, n\}, i \neq j$. Now $a_{i} \notin M_{i}$ but $a_{j} \in M_{i}$. So $a_{i}$ and $a_{j}$ are distinct. Note that $a_{i} \in M_{1}$ for $2 \leq i \leq n$ and $a_{1} \in M_{2}$ but $a_{n+1} \notin M_{1} \cup M_{2}$. Hence $a_{n+1} \notin\left\{a_{1}, a_{2}, \ldots, a_{n}\right\}$. Let $1 \leq i, j \leq n, i \neq j$. Since $n \geq 4, n \geq 3$. Hence there exists $k \in\{1,2, \ldots, n\} \backslash\{i, j\}$. Note that both $a_{i}$ and $a_{j}$ are in $M_{k}$. It is clear that $a_{n+1}$ and $a_{i}$ are in $M_{n}$ for $1 \leq i \leq n-1$. Observe that both $a_{n+1}$ and $a_{n}$ belong to $M_{n-1}$. Hence the subgraph of $(G(R))^{c}$ induced on $\left\{a_{1}, a_{2}, \ldots, a_{n+1}\right\}$ is a clique. This is in contradiction to the assumption that $\omega\left((G(R))^{c}\right) \leq n$. Therefore, $|\operatorname{Max}(R)| \leq n-1$.

Lemma 3. Let $R$ be a semiquasilocal ring which is not quasilocal. If there exists $k \in \mathbf{N}$ such that $|M \backslash J(R)| \leq k$ for all $M \in \operatorname{Max}(R)$, then $R$ is finite.

Proof. Let $\left\{M_{1}, M_{2}, \ldots, M_{n}\right\}$ be the set of all maximal ideals of $R$. Note that $n \geq 2$. In view of Lemma 1 , to prove $R$ is finite, it is enough to prove that $N U(R)$ is finite. Observe that $N U(R)=$ $J(R) \cup\left(\bigcup_{i \in\{1,2, \ldots, n\}}\left(M_{i} \backslash J(R)\right)\right)$. By hypothesis, $\left|M_{i} \backslash J(R)\right| \leq k$ for $i=1,2, \ldots, n$. Thus that $N U(R)$ is finite will follow if we show that $J(R)$ is finite. We claim that $|J(R)| \leq k$. Let $m \in$ $M_{1} \backslash J(R)$. Note that $\{m+j \mid j \in J(R)\} \subseteq M_{1} \backslash J(R)$ and so $|\{m+j \mid j \in J(R)\}| \leq\left|M_{1} \backslash J(R)\right| \leq k$. Hence $|J(R)|=\mid\{m+j \mid$ $j \in J(R)\} \mid \leq k$. This proves that $N U(R)$ is finite and so $R$ is a finite ring.

Corollary 4. Let $n \geq 4$. Let $R$ be a ring with at least two maximal ideals. If $\omega\left((G(R))^{c}\right) \leq n$, then $R$ is finite.

Proof. By assumption, $\omega\left((G(R))^{c}\right) \leq n$. Hence by Lemma 2, $|\operatorname{Max}(R)| \leq n-1$. Moreover, since for any maximal ideal $M$ of $R$, the subgraph of $(G(R))^{c}$ induced on $M \backslash J(R)$ is a clique, it follows that $|M \backslash J(R)| \leq n$ for any $M \in \operatorname{Max}(R)$. Therefore, we obtain from Lemma 3 that $R$ is finite.

Lemma 5. Let $\left\{M_{1}, M_{2}\right\}$ be the set of all maximal ideals of a ring $R$. Then $(G(R))^{c}$ has exactly two components and if $\left|M_{i}\right|$ $J(R) \mid \leq 4$ for $i=1,2$, then $(G(R))^{c}$ is planar.

Proof. Let $g_{1}$ (resp., $g_{2}$ ) be the subgraph of $(G(R))^{c}$ induced on $M_{1} \backslash M_{2}$ (resp., $M_{2} \backslash M_{1}$ ). Let $V_{1}=M_{1} \backslash M_{2}$ and $V_{2}=$ $M_{2} \backslash M_{1}$. Observe that $V_{1} \neq \emptyset, V_{2} \neq \emptyset$ and $V_{1} \cap V_{2}=\emptyset$. For any $a \in V_{1}$ and $b \in V_{2}, R a+R b=R$. Hence there is no edge of $(G(R))^{c}$ whose one end vertex is in $V_{1}$ and the other is in $V_{2}$. Therefore, there is no path in $(G(R))^{c}$ between any $a \in V_{1}$ and $b \in V_{2}$. Moreover, it is clear that $g_{1}$ and $g_{2}$ are complete. This proves that $g_{1}$ and $g_{2}$ are the only components of $(G(R))^{c}$. It is well known that any complete graph on at most four vertices is planar. Hence if $\left|M_{i} \backslash J(R)\right| \leq 4$ for $i=1,2$, it follows that $(G(R))^{c}$ is planar.

Lemma 6. Let $(R, M)$ be a quasilocal ring with $M \neq(0)$. If $n \in \mathbf{N}$ with $n \geq 2$ is such that $|M| \leq n$, then $|R / M| \leq n$. In particular, if $|R|=8$, then $|M|=4$.
Proof. Now $|M| \leq n$. We assert that $|R / M| \leq n$. Suppose that $|R / M| \geq n+1$. Let $\left\{0+M, 1+M, r_{1}+M, r_{2}+\right.$ $\left.M, \ldots, r_{n-1}+M\right\} \subseteq R / M$. Let $m \in M \backslash(0)$. Note that for any quasilocal ring $(R, M)$, if $a, b \in R$ with $a-b \notin M$, then for any $m \in M \backslash(0), a m \neq b m$. Therefore, it follows that $\left\{0, m, r_{1} m, r_{2} m, \ldots, r_{n-1} m\right\} \subseteq M$. Hence $|M| \geq n+1$. This is a contradiction to the hypothesis that $|M| \leq n$. Therefore, $|R / M| \leq n$.

Let $|R|=8$. Now $8=|R|=|M||R / M|$. Hence $|M|=2^{i}$ for some $i, 1 \leq i<3$. If $i=1$, then we obtain $|M|=2$ and so it follows from the previous paragraph that $|R / M|=2$. Hence $|R|=|M||R / M|=2 \times 2=4$. This is a contradiction. Therefore, $|M|=4$.

Remark 7. Let $(R, M)$ be a finite local ring which is not a field. If $|R|=9$, then $|M|=3$.

Proof. Now $|R|=9=|M||R / M|$. As $|M| \geq 2$ and $|R / M| \geq 2$, it follows that $|M|=3=|R / M|$.

Observation 1. Let $G=(V, E)$ be a graph. If $G$ contains $K_{6}$ as a subgraph, then $G$ neither satisfies $K u_{1}$ nor satisfies $K u_{2}$.

Proof. It is obvious.

Lemma 8. Let $R$ be a ring with at least four maximal ideals. Then $(G(R))^{c}$ neither satisfies $K u_{1}$ nor satisfies $K u_{2}$.

Proof. By hypothesis, $R$ has at least four maximal ideals. Let $\left\{M_{1}, M_{2}, M_{3}, M_{4}\right\} \subseteq \operatorname{Max}(R)$. Consider the elements $a, b, c$, $d, e, f$ defined by

$$
\begin{aligned}
& a \in\left(M_{1} \cap M_{2}\right) \backslash\left(M_{3} \cup M_{4}\right) \\
& b \in\left(M_{1} \cap M_{3}\right) \backslash\left(M_{2} \cup M_{4}\right) \\
& c \in\left(M_{1} \cap M_{4}\right) \backslash\left(M_{2} \cup M_{3}\right) \\
& d \in\left(M_{1} \cap M_{2} \cap M_{3}\right) \backslash M_{4} \\
& e \in\left(M_{1} \cap M_{2} \cap M_{4}\right) \backslash M_{3} \\
& f \in\left(M_{1} \cap M_{3} \cap M_{4}\right) \backslash M_{2} .
\end{aligned}
$$

It is clear that the above elements $a, b, c, d, e, f$ are distinct. As they all belong to $M_{1} \backslash J(R)$, it follows that the subgraph of $(G(R))^{c}$ induced on $\{a, b, c, d, e, f\}$ is a clique. Hence $(G(R))^{c}$ contains $K_{6}$ as a subgraph. Therefore, by Observation 1 , we obtain that $(G(R))^{c}$ neither satisfies $K u_{1}$ nor satisfies $K u_{2}$.

Lemma 9. Let $R$ be a ring with at least two maximal ideals. If $(G(R))^{c}$ satisfies $K u_{1}$ or $K u_{2}$, then $R$ is finite.

Proof. Assume that $(G(R))^{c}$ satisfies either $K u_{1}$ or $K u_{2}$. Then it is clear that $\omega\left((G(R))^{c}\right) \leq 5$. Therefore, we obtain from Corollary 4 that $R$ is a finite ring.

Lemma 10. Let $R$ be a ring with at least two maximal ideals. If $(G(R))^{c}$ satisfies $K u_{1}$ or $K u_{2}$, then $R$ is isomorphic to the direct product of finite local rings $\left(R_{i}, M_{i}\right)(i=1,2, \ldots, n)$ with $2 \leq$ $n \leq 3$.

Proof. We know from Lemma 8 that $|\operatorname{Max}(R)| \leq 3$ and from Lemma 9 that $R$ is finite. Since any finite ring is isomorphic to 
the direct product of finite local rings and as $2 \leq|\operatorname{Max}(R)| \leq$ 3 , it follows that either $R \approx R_{1} \times R_{2}$ or $R \approx R_{1} \times R_{2} \times R_{3}$ as rings, where $\left(R_{i}, M_{i}\right)$ is a finite local ring for each $i \in\{1,2,3\}$.

\section{Characterization of Rings $R$ with Exactly Two Maximal Ideals Such That $(G(R))^{c}$ Is Planar}

The aim of this section is to characterize rings $R$ with $|\operatorname{Max}(R)|=2$ such that $(G(R))^{c}$ is planar.

Lemma 11. Let $R=F_{1} \times F_{2}$, where $F_{1}$ and $F_{2}$ are fields. If $(G(R))^{c}$ satisfies $K u_{1}$ or $K u_{2}$, then $\left|F_{i}\right| \leq 5$ for $i=1,2$.

Proof. Now $R=F_{1} \times F_{2}$. Note that $R$ has exactly two maximal ideals $M_{1}$ and $M_{2}$, where $M_{1}=(0) \times F_{2}$ and $M_{2}=F_{1} \times(0)$. Observe that $J(R)=\{(0,0)\}$. As $(G(R))^{c}$ satisfies either $K u_{1}$ or $K u_{2},\left|M_{i} \backslash J(R)\right| \leq 5$ for $i=1,2$. Hence $\left|M_{i}\right| \leq 6$ for $i=1,2$. Therefore, $\left|F_{i}\right| \leq 6$ for $i=1,2$. As there is no field with exactly 6 elements, it follows that $\left|F_{i}\right| \leq 5$ for $i=1,2$.

Lemma 12. Let $R=F_{1} \times F_{2}$, where $F_{1}$ and $F_{2}$ are fields. If $\left|F_{i}\right| \leq 5$ for $i=1,2$, then $(G(R))^{c}$ is planar.

Proof. Note that $M_{1}=(0) \times F_{2}$ and $M_{2}=F_{1} \times(0)$ are the only maximal ideals of $R$. Now $\left|M_{1} \backslash M_{2}\right|=\left|(0) \times F_{2}^{*}\right| \leq 4$, and $\left|M_{2} \backslash M_{1}\right|=\left|F_{1}^{*} \times(0)\right| \leq 4$. So we obtain from Lemma 5 that $(G(R))^{c}$ is planar.

Lemma 13. Let $R=F_{1} \times R_{2}$, where $F_{1}$ is a field and $\left(R_{2}, M_{2}\right)$ is a quasilocal ring which is not a field. If $(G(R))^{c}$ satisfies $K u_{1}$ or $K u_{2}$, then $\left|F_{1}\right| \leq 3$ and $\left|R_{2}\right| \leq 8$. And in the case $\left|F_{1}\right|=3$, $R_{2}$ must be a local ring with $\left|R_{2}\right|=4$.

Proof. Note that $N_{1}=(0) \times R_{2}$ and $N_{2}=F_{1} \times M_{2}$ are the only maximal ideals of $R$. As $(G(R))^{c}$ satisfies $K u_{1}$ or $K u_{2}$, $\left|N_{1} \backslash N_{2}\right| \leq 5$ and $\left|N_{2} \backslash N_{1}\right| \leq 5$. Now $\left|N_{1} \backslash N_{2}\right| \leq 5$ implies that $\left|(0) \times\left(R_{2} \backslash M_{2}\right)\right| \leq 5$. Hence $\left|R_{2} \backslash M_{2}\right| \leq 5$. Therefore, $\left|M_{2}\right| \leq 5$ and so $\left|R_{2}\right|=\left|M_{2}\right|+\left|R_{2} \backslash M_{2}\right| \leq 10$. Now $\left|N_{2} \backslash N_{1}\right| \leq$ 5 implies that $\left|F_{1}^{*} \times M_{2}\right| \leq 5$. As $\left|M_{2}\right| \geq 2$, we obtain that $2\left|F_{1}^{*}\right| \leq\left|F_{1}^{*}\right|\left|M_{2}\right| \leq 5$. Therefore, $\left|F_{1}^{*}\right| \leq 2$ and this implies that $\left|F_{1}\right| \leq 3$. Now $\left(R_{2}, M_{2}\right)$ is a local ring which is not a field and $\left|R_{2}\right| \leq 10$. And as the order of any finite local ring is a power of a prime, it follows that $\left|R_{2}\right| \in\{4,8,9\}$. Suppose that $\left|R_{2}\right|=9$ and $\left|F_{1}\right| \in\{2,3\}$. Then $\left|R_{2} \backslash M_{2}\right|=6$ by Remark 7 . Hence $\left|N_{1} \backslash N_{2}\right|=\left|(0) \times\left(R_{2} \backslash M_{2}\right)\right|=6$. Since the subgraph of $(G(R))^{c}$ induced on $N_{1} \backslash N_{2}$ is $K_{6}$, it follows that $(G(R))^{c}$ neither satisfies $K u_{1}$ nor satisfies $K u_{2}$. Hence $\left|R_{2}\right|=9$ is not possible. Suppose that $\left|R_{2}\right|=8$ and $\left|F_{1}\right|=3$. Then $\left|M_{2}\right|=4$ by Lemma 6 . Note that $\left|N_{2} \backslash N_{1}\right|=\left|F_{1}^{*} \times M_{2}\right|=\left|F_{1}^{*}\right|\left|M_{2}\right|=$ $2 \times 4=8$. In this case the subgraph of $(G(R))^{c}$ induced on $N_{2} \backslash N_{1}$ is $K_{8}$. This is impossible as $(G(R))^{c}$ satisfies $K u_{1}$ or $K u_{2}$. This proves that if $(G(R))^{c}$ satisfies $K u_{1}$ or $K u_{2}$, then either $\left|F_{1}\right|=2$ and $\left|R_{2}\right| \in\{4,8\}$ or $\left|F_{1}\right|=3$ and $\left|R_{2}\right|=4$.

Lemma 14. Let $R=F_{1} \times R_{2}$, where $F_{1}$ is a field with $\left|F_{1}\right|=2$ and $\left(R_{2}, M_{2}\right)$ is a quasilocal ring which is not a field with $\left|R_{2}\right|=$ 4. Then $(G(R))^{c}$ is planar.
Proof. Note that $N_{1}=(0) \times R_{2}$ and $N_{2}=F_{1} \times M_{2}$ are the only maximal ideals of $R$. Now $\left|N_{1} \backslash N_{2}\right|=\left|(0) \times\left(R_{2} \backslash M_{2}\right)\right|=\mid R_{2} \backslash$ $M_{2} \mid=2$ and $\left|N_{2} \backslash N_{1}\right|=\left|F_{1}^{*} \times M_{2}\right|=\left|F_{1}^{*}\right|\left|M_{2}\right|=\left|M_{2}\right|=2$. Hence by Lemma 5, we obtain that $(G(R))^{c}$ is planar.

Lemma 15. Let $R=F_{1} \times R_{2}$, where $F_{1}$ is a field with $\left|F_{1}\right|=2$ and $\left(R_{2}, M_{2}\right)$ is a quasilocal ring which is not a field with $\left|R_{2}\right|=$ 8. Then $(G(R))^{c}$ is planar.

Proof. Note that $\operatorname{Max}(R)=\left\{N_{1}=(0) \times R_{2}, N_{2}=F_{1} \times M_{2}\right\}$. Now $\left|N_{1} \backslash N_{2}\right|=\left|(0) \times\left(R_{2} \backslash M_{2}\right)\right|=\left|R_{2} \backslash M_{2}\right|=4$ and $\left|N_{2} \backslash N_{1}\right|=\left|F_{1}^{*} \times M_{2}\right|=\left|F_{1}^{*}\right|\left|M_{2}\right|=\left|M_{2}\right|=4$. So by Lemma 5, it follows that $(G(R))^{c}$ is planar.

Lemma 16. Let $R=F_{1} \times R_{2}$, where $F_{1}$ is a field with $\left|F_{1}\right|=3$ and $\left(R_{2}, M_{2}\right)$ is a quasilocal ring which is not a field with $\left|R_{2}\right|=$ 4.Then $(G(R))^{c}$ is planar.

Proof. Note that $\operatorname{Max}(R)=\left\{N_{1}=(0) \times R_{2}, N_{2}=F_{1} \times M_{2}\right\}$. Now $\left|N_{1} \backslash N_{2}\right|=\left|(0) \times\left(R_{2} \backslash M_{2}\right)\right|=\left|R_{2} \backslash M_{2}\right|=2$ and $\left|N_{2} \backslash N_{1}\right|=\left|F_{1}^{*} \times M_{2}\right|=\left|F_{1}^{*}\right|\left|M_{2}\right|=2 \times 2=4$. So by Lemma 5 , we obtain that $(G(R))^{c}$ is planar.

Lemma 17. Let $R=R_{1} \times R_{2}$, where $\left(R_{i}, M_{i}\right)$ is a quasilocal ring which is not a field for $i=1,2$. If $(G(R))^{c}$ satisfies $K u_{1}$ or $K u_{2}$, then $\left|R_{1}\right|=\left|R_{2}\right|=4$.

Proof. Note that $N_{1}=R_{1} \times M_{2}$ and $N_{2}=M_{1} \times R_{2}$ are the only maximal ideals of $R$. As $(G(R))^{c}$ satisfies $K u_{1}$ or $K u_{2}$, $\left|N_{i} \backslash J(R)\right| \leq 5$ for $i=1,2$. Now, $\left|N_{1} \backslash N_{2}\right|=\mid\left(R_{1} \backslash M_{1}\right) \times$ $M_{2} \mid \leq 5$ and as $\left|R_{1} \backslash M_{1}\right| \geq 2$ and $\left|M_{2}\right| \geq 2$, we obtain that $4 \leq\left|R_{1} \backslash M_{1}\right|\left|M_{2}\right| \leq 5$. This implies that $\left|R_{1} \backslash M_{1}\right|=2=\left|M_{2}\right|$. Hence $\left|M_{1}\right|=2$ and so $\left|R_{1}\right|=4$. Similarly from $\left|N_{2} \backslash N_{1}\right| \leq 5$, it follows that $\left|R_{2}\right|=4$.

Lemma 18. Let $R=R_{1} \times R_{2}$, where $\left(R_{i}, M_{i}\right)$ is a quasilocal ring which is not a field with $\left|R_{i}\right|=4$ for $i=1,2$. Then $(G(R))^{c}$ is planar.

Proof. Note that $\operatorname{Max}(R)=\left\{N_{1}=R_{1} \times M_{2}, N_{2}=M_{1} \times R_{2}\right\}$. Now $\left|N_{1} \backslash N_{2}\right|=\left|\left(R_{1} \backslash M_{1}\right) \times M_{2}\right|=2 \times 2=4$ and $\left|N_{2} \backslash N_{1}\right|=$ $\left|M_{1} \times\left(R_{2} \backslash M_{2}\right)\right|=2 \times 2=4$. Hence it follows from Lemma 5 that $(G(R))^{c}$ is planar.

The following theorem determines necessary and sufficient conditions for $(G(R))^{c}$ to be planar, where $R$ is a ring with $|\operatorname{Max}(R)|=2$.

Theorem 19. Let $R$ be a ring with exactly two maximal ideals. Then the following statements are equivalent.

(1) $(G(R))^{c}$ satisfies $K u_{1}$.

(2) $R$ is isomorphic to one of the following rings.

(a) $F_{1} \times F_{2}$, where $F_{1}$ and $F_{2}$ are fields with $\left|F_{i}\right| \leq 5$ for $i=1,2$.

(b) $F_{1} \times R_{2}$, where $F_{1}$ is a field with $\left|F_{1}\right| \leq 3$ and $\left(R_{2}, M_{2}\right)$ is a local ring which is not a field and 
$\left|R_{2}\right| \leq 8$ and if $\left|F_{1}\right|=3$, then $R_{2}$ must be a local ring with $\left|R_{2}\right|=4$.

(c) $R_{1} \times R_{2}$, where both $R_{1}$ and $R_{2}$ are local rings which are not fields with $\left|R_{i}\right|=4$ for $i=1,2$.

(3) $(G(R))^{c}$ satisfies $K u_{2}$.

(4) $(G(R))^{c}$ is planar.

Proof. (1) $\Rightarrow$ (2) (resp., (3) $\Rightarrow$ (2))

Now $R$ has exactly two maximal ideals and $(G(R))^{c}$ satisfies $K u_{1}$ (respectively, $K u_{2}$ ). Hence from Lemma 10 we obtain that $R \approx R_{1} \times R_{2}$ as rings, where $\left(R_{i}, M_{i}\right)$ is a quasilocal ring for $i=1,2$. If both $R_{1}$ and $R_{2}$ are fields, then Lemma 11 implies that $R$ is isomorphic to the ring given in (a). If $R_{1}$ is a field and $R_{2}$ is not a field, then from Lemma 13, we obtain that $R$ is isomorphic to the ring described in (b). If both $R_{1}$ and $R_{2}$ are not fields, then it follows from Lemma 17 that $R$ is isomorphic to the ring given in (c).

$(2) \Rightarrow(4)$. This follows immediately from Lemmas 12,14 , 15,16 , and 18 .

$(4) \Rightarrow(1)$ and $(4) \Rightarrow(3)$. Indeed it follows from Kuratowski's theorem [8, Theorem 5.9] that $(G(R))^{c}$ satisfies both $K u_{1}^{*}$ and $K u_{2}^{*}$.

\section{Characterization of Rings $R$ with Exactly Three Maximal Ideals Such That $(G(R))^{c}$ Is Planar}

The main result of this section is Theorem 24 which characterizes rings $R$ with $|\operatorname{Max}(R)|=3$ such that $(G(R))^{c}$ is planar.

Lemma 20. Let $R=R_{1} \times R_{2} \times R_{3}$, where $\left(R_{i}, M_{i}\right)$ is a quasilocal ring for $i=1,2$, 3. If at least one $R_{i}$ is not a field, then $(G(R))^{c}$ neither satisfies $K u_{1}$ nor satisfies $K u_{2}$.

Proof. Without loss of generality we may assume that $\left(R_{1}, M_{1}\right)$ is not a field. Let $a \in M_{1} \backslash\{0\}$. Then the subgraph of $(G(R))^{c}$ induced on $\{(0,1,1),(a, 0,1),(a, 1,0),(0,1,0),(a, 1,1),(0,0,1)\} \quad$ is $\quad$ a clique. Thus $(G(R))^{c}$ contains $K_{6}$ as a subgraph. Therefore, $(G(R))^{c}$ neither satisfies $K u_{1}$ nor satisfies $K u_{2}$.

Lemma 21. Let $R=F_{1} \times F_{2} \times F_{3}$, where $F_{i}$ is a field for $i=1,2,3$. If $(G(R))^{c}$ satisfies $K u_{1}$, then $F_{i}=\mathbf{Z}_{2}$ for $i=1,2,3$.

Proof. Note that $\operatorname{Max}(R)=\left\{M_{1}=F_{1} \times F_{2} \times(0), M_{2}=(0) \times\right.$ $\left.F_{2} \times F_{3}, M_{3}=F_{1} \times(0) \times F_{3}\right\}$ and $J(R)=\{(0,0,0)\}$. As $(G(R))^{c}$ satisfies $K u_{1},\left|M_{1} \backslash J(R)\right| \leq 4$. This implies that $\left|F_{1} \times F_{2} \times(0)\right|-$ $1 \leq 4$. So $\left|F_{1}\right|\left|F_{2}\right| \leq 5$. It follows that $2 \times 2=4 \leq\left|F_{1}\right|\left|F_{2}\right| \leq 5$. Hence $\left|F_{i}\right|=2$ for $i=1,2$. Similarly we obtain that $\left|F_{3}\right|=2$. Therefore, $R \approx \mathbf{Z}_{2} \times \mathbf{Z}_{2} \times \mathbf{Z}_{2}$ as rings.

Lemma 22. Let $R=F_{1} \times F_{2} \times F_{3}$, where $F_{i}$ is a field for $i=$ $1,2,3$. If $(G(R))^{c}$ satisfies $K u_{2}$, then $F_{i}=\mathbf{Z}_{2}$ for $i=1,2,3$.

Proof. Assume that $(G(R))^{c}$ satisfies $K u_{2}$. We assert that $\left|F_{i}\right|=$ 2 for each $i \in\{1,2,3\}$. Suppose that $\left|F_{i}\right| \geq 3$ for some $i \in\{1,2,3\}$. Without loss of generality we may assume that $\left|F_{1}\right| \geq 3$. Let $\alpha \in F_{1} \backslash\{0,1\}$. Note that $\operatorname{Max}(R)=$
$\left\{M_{1}=F_{1} \times F_{2} \times(0), M_{2}=(0) \times F_{2} \times F_{3}, M_{3}=F_{1} \times\right.$ $\left.(0) \times F_{3}\right\}$. Let $V_{1}=\{(0,0,1),(\alpha, 0,0),(1,0,0)\}$ and $V_{2}=$ $\{(\alpha, 0,1),(0,1,0),(1,0,1)\}$. Note that $V_{1} \cap V_{2}=\emptyset$ and each vertex of $V_{1}$ is adjacent to every vertex of $V_{2}$ in $(G(R))^{c}$. Hence the subgraph of $(G(R))^{c}$ induced on $V_{1} \cup V_{2}$ contains $K_{3,3}$ as a subgraph. This is in contradiction to the assumption that $(G(R))^{c}$ satisfies $K u_{2}$. Therefore, $\left|F_{i}\right|=2$ for $i=1,2,3$.

Lemma 23. If $R \approx \mathbf{Z}_{2} \times \mathbf{Z}_{2} \times \mathbf{Z}_{2}$, then $(G(R))^{c}$ is planar.

Proof. Note that $(G(R))^{c}$ is the union of cycles:

$$
\begin{aligned}
& C_{1}:(0,1,1)-(0,0,1)-(1,0,1)-(1,0,0)-(1,1,0)- \\
& (0,1,0)-(0,1,1), \\
& C_{2}:(0,0,1)-(1,0,0)-(0,1,0)-(0,0,1) .
\end{aligned}
$$

Observe that $C_{1}$ is a cycle of length 6 and it can be represented by means of a hexagon and $C_{2}$ is a cycle of length 3 . Note that each edge of $C_{2}$ is a diagonal of the hexagon representing $C_{1}$ and hence it can be represented by means of a triangle which can be drawn inside the hexagon without any crossing over of the edges. This shows that $(G(R))^{c}$ is planar.

Theorem 24. Let $R$ be a ring with exactly three maximal ideals. Then the following statements are equivalent.

(1) $(G(R))^{c}$ satisfies $K u_{1}$.

(2) $R \approx \mathbf{Z}_{2} \times \mathbf{Z}_{2} \times \mathbf{Z}_{2}$ as rings.

(3) $(G(R))^{c}$ satisfies $K u_{2}$.

(4) $(G(R))^{c}$ is planar.

Proof. (1) $\Rightarrow$ (2) (resp., (3) $\Rightarrow$ (2)).

Now by hypothesis $R$ has exactly three maximal ideals and $(G(R))^{c}$ satisfies $K u_{1}$ (respectively, $K u_{2}$ ). Hence we obtain from Lemma 10 that $R \approx R_{1} \times R_{2} \times R_{3}$ as rings, where $\left(R_{i}, M_{i}\right)$ is a quasilocal ring for $i=1,2,3$. Now it follows from Lemmas 20 and 21 (respectively, Lemmas 20 and 22) that $R_{i}=\mathbf{Z}_{2}$ for $i=1,2,3$. Therefore, $R \approx \mathbf{Z}_{2} \times \mathbf{Z}_{2} \times \mathbf{Z}_{2}$ as rings.

(2) $\Rightarrow(4)$

This follows from Lemma 23 .

(4) $\Rightarrow$ (1) and (4) $\Rightarrow$ (3).

It follows from Kuratowski's theorem [8, Theorem 5.9] that $(G(R))^{c}$ satisfies both $K u_{1}^{*}$ and $K u_{2}^{*}$.

\section{Characterization of Rings $R$ with at Least Two Maximal Ideals Such That $(\Omega(R))^{c}$ Is Planar}

The aim of this section is to characterize rings $R$ with at least two maximal ideals such that $(\Omega(R))^{c}$ is planar. If $(\Omega(R))^{c}$ is planar, then $(G(R))^{c}$ being a subgraph of $(\Omega(R))^{c}$ must also be planar. Hence we obtain from Lemma 10 that $R$ is isomorphic to the direct product of either two or three finite local rings.

Lemma 25. Let $R=F_{1} \times F_{2}$, where $F_{1}$ and $F_{1}$ are fields. If at least one $F_{i}$ contains five elements for some $i \in\{1,2\}$, then $(\Omega(R))^{c}$ does not satisfy $\mathrm{Ku} u_{1}$. 
Proof. Note that $M_{1}=F_{1} \times(0)$ and $M_{2}=(0) \times F_{2}$ are the only maximal ideals of $R$. Without loss of generality we may assume that $\left|F_{1}\right| \geq 5$. Now $\left|M_{1}\right|=\left|F_{1} \times(0)\right|=\left|F_{1}\right| \geq 5$. It is clear that the subgraph of $(\Omega(R))^{c}$ induced on $M_{1}$ is a clique. So it follows that $(\Omega(R))^{c}$ contains $K_{5}$ as a subgraph. Therefore, $(\Omega(R))^{c}$ does not satisfy $K u_{1}$.

Lemma 26. Let $\left\{M_{1}, M_{2}\right\}$ be the set of all maximal ideals of a ring $R$ with $\left|M_{i}\right| \leq 4$ for $i=1$, 2. If $M_{1} \cap M_{2}=(0)$, then $(\Omega(R))^{c}$ is planar.

Proof. Note that the vertex set of $(\Omega(R))^{c}=\left(M_{1} \backslash(0)\right) \cup\left(M_{2} \backslash\right.$ $(0)) \cup(0) \cup U(R)$. Let $V_{i}=M_{i} \backslash(0)$ for $i=1,2, V_{3}=(0)$, $V_{4}=U(R)$. It is clear that $V_{i} \cap V_{j}=\emptyset$ for all distinct $i, j \in$ $\{1,2,3,4\}$. Observe that the subgraph of $(\Omega(R))^{c}$ induced on $V_{i}$ is a complete graph on at most three vertices for $i=1,2$ and each vertex of $V_{4}$ is an isolated vertex. The vertex 0 is adjacent to every vertex of $V_{1} \cup V_{2}$ and $\left|V_{1} \cup V_{2}\right| \leq 6$. Now it is clear that $(\Omega(R))^{c}$ can be drawn in a plane in such a way that there is no crossing over of the edges. This proves that $(\Omega(R))^{c}$ is planar.

Corollary 27. Let $R=F_{1} \times F_{2}$, where $F_{1}$ and $F_{2}$ are fields with $\left|F_{i}\right| \leq 4$ for $i=1,2$. Then $(\Omega(R))^{c}$ is planar.

Proof. Observe that $M_{1}=F_{1} \times(0)$ and $M_{2}=(0) \times F_{2}$ are the only maximal ideals of $R$ with $\left|M_{i}\right|=\left|F_{i}\right| \leq 4$ for $i=1,2$ and $M_{1} \cap M_{2}=\{(0,0)\}$. Hence it follows immediately from Lemma 26 that $(\Omega(R))^{c}$ is planar.

Lemma 28. Let $R=R_{1} \times R_{2}$, where $\left(R_{i}, M_{i}\right)$ is a quasilocal ring for $i=1,2$ and $\left(R_{2}, M_{2}\right)$ is not a field. If $(\Omega(R))^{c}$ satisfies $K u_{1}$ or $K u_{2}$, then $\left|R_{1}\right|=2$ and $\left|R_{2}\right|=4$.

Proof. Note that $\left\{N_{1}=R_{1} \times M_{2}, N_{2}=M_{1} \times R_{2}\right\}$ is the set of all maximal ideals of $R$. If $(\Omega(R))^{c}$ satisfies $K u_{1}$ or $K u_{2}$, then $\left|N_{i}\right| \leq 5$ for $i=1,2$. Since $R_{2}$ is not a field, $\left|M_{2}\right| \geq 2$. Thus if $\left|R_{1}\right| \geq 3$, then $\left|N_{1}\right|=\left|R_{1}\right|\left|M_{2}\right| \geq 6$. This is impossible. Therefore, $\left|R_{1}\right|=2$. Thus $M_{1}=(0)$ and so $\left|M_{1}\right|=1$. We next verify that $\left|R_{2}\right|=4$. Since $R_{2}$ is not a field, it follows that $\left|R_{2}\right| \geq 4$. As $\left|N_{2}\right| \leq 5$, we obtain that $\left|M_{1} \times R_{2}\right|=\left|R_{2}\right| \leq 5$. Therefore, $\left|R_{2}\right|=4$.

Lemma 29. Let $R=F_{1} \times R_{2}$, where $F_{1}$ is a field with $\left|F_{1}\right|=2$ and $\left(R_{2}, M_{2}\right)$ is a quasilocal ring which is not a field with $\left|R_{2}\right|=$ 4. Then $(\Omega(R))^{c}$ is planar.

Proof. Now $F_{1}=\{0,1\}$. As $\left|R_{2}\right|=4$, it follows that $\left|M_{2}\right|=2$. Let $M_{2}=\{0, a\}$. Note that $R_{2}=\{0,1, a, 1+a\}$. Observe that the vertex set of $(\Omega(R))^{c}=R=V_{1} \cup V_{2} \cup V_{3}$, where $V_{1}=$ $\{(1,0),(1, a),(0, a),(0,0)\}, V_{2}=\{(0,1),(0,1+a),(0, a),(0,0)\}$, and $V_{3}=\{(1,1),(1,1+a)\}$. Note that the subgraph $g_{i}$ of $(\Omega(R))^{c}$ induced on $V_{i}$ is complete for $i=1,2$. Each vertex of $V_{3}$ is an isolated vertex. Thus $(\Omega(R))^{c}$ is the union of $g_{1}, g_{2}$ and two isolated vertices. Since $K_{4}$ is planar and as the edge $(0,0)-(0, a)$ is the only edge which is common to both $g_{1}$ and $g_{2},(\Omega(R))^{c}$ can be drawn in a plane in such a way that there is no crossing over of the edges. This proves that $(\Omega(R))^{c}$ is planar.
Lemma 30. Let $R=R_{1} \times R_{2} \times R_{3}$, where $\left(R_{i}, M_{i}\right)$ is a quasilocal ring for $i=1,2$, 3. If $(\Omega(R))^{c}$ satisfies $K u_{1}$ or $K u_{2}$, then $\left|R_{i}\right|=2$ for $i=1,2,3$.

Proof. Note that $R$ is a ring with exactly three maximal ideals. If $(\Omega(R))^{c}$ satisfies $K u_{1}$ (resp., $\left.K u_{2}\right)$, then $(G(R))^{c}$ satisfies $K u_{1}$ (resp., $K u_{2}$ ). Hence it follows from (1) $\Rightarrow$ (2) (resp., $(3) \Rightarrow(2))$ of Theorem 24 that $\left|R_{i}\right|=2$ for $i=1,2,3$.

Lemma 31. If $R \approx \mathbf{Z}_{2} \times \mathbf{Z}_{2} \times \mathbf{Z}_{2}$, then $(\Omega(R))^{c}$ is planar.

Proof. Observe that $V\left((\Omega(R))^{c}\right)=V\left((G(R))^{c}\right) \quad U$ $\{(0,0,0),(1,1,1)\}$. It was already noted in the proof of Lemma 23 that $(G(R))^{c}$ is the union of the cycles $C_{1}$ : $(0,1,1)-(0,0,1)-(1,0,1)-(1,0,0)-(1,1,0)-(0,1,0)-(0,1,1)$ and $C_{2}:(0,0,1)-(1,0,0)-(0,1,0)-(0,0,1)$. Note that $C_{1}$ is a cycle of length 6 and it can be represented by means of a hexagon. The vertex $(0,0,0)$ is adjacent to all the six vertices of $C_{1}$ in $(\Omega(R))^{c}$. The vertex $(0,0,0)$ can be plotted inside the hexagon representing $C_{1}$ and it can be joined to all the vertices of $C_{1}$ without any crossing over of the edges. The edges of $C_{2}$, each of which is a diagonal of the hexagon representing $C_{1}$, can be drawn outside the hexagon in such a way that there is no crossing over of the edges. The vertex $(1,1,1)$ is an isolated vertex of $(\Omega(R))^{c}$. This proves that $(\Omega(R))^{c}$ is planar.

Theorem 32. Let $R$ be a ring with exactly two maximal ideals. Then the following statements are equivalent.

(1) $(\Omega(R))^{c}$ satisfies $K u_{1}$ and $K u_{2}$.

(2) $(\Omega(R))^{c}$ satisfies $K u_{1}$.

(3) $R$ is isomorphic to one of the following rings:

(a) $F_{1} \times F_{2}$, where $F_{1}$ and $F_{2}$ are fields with $\left|F_{i}\right| \leq 4$ for $i=1,2$;

(b) $F_{1} \times R_{2}$, where $F_{1}$ is a field with $\left|F_{1}\right|=2$ and $\left(R_{2}, M_{2}\right)$ is a local ring which is not a field and $\left|R_{2}\right|=4$.

(4) $(\Omega(R))^{c}$ is planar.

Proof. (1) $\Rightarrow(2)$ This is clear.

$(2) \Rightarrow(3)$

Now by hypothesis $R$ has exactly two maximal ideals and $(\Omega(R))^{c}$ satisfies $K u_{1}$. As $(G(R))^{c}$ is a subgraph of $(\Omega(R))^{c}$, it follows that $(G(R))^{c}$ satisfies $K u_{1}$. Hence from Lemma 10 it follows that $R \approx R_{1} \times R_{2}$ as rings, where $\left(R_{i}, M_{i}\right)$ is a local ring for $i=1,2$. Now if both $R_{1}$ and $R_{2}$ are fields, then it follows from Lemma 25 that $R$ is isomorphic to the ring given in (a). If either $R_{1}$ or $R_{2}$ is not a field, then it follows from Lemma 28 that $R$ is isomorphic to the ring given in (b).

(3) $\Rightarrow(4)$.

This follows from Corollary 27 and Lemma 29:

(4) $\Rightarrow(1)$.

It follows from Kuratowski's theorem [8, Theorem 5.9] that $(\Omega(R))^{c}$ satisfies both $K u_{1}^{*}$ and $K u_{2}^{*}$.

Lemma 33. Let $R=F_{1} \times F_{2}$, where $F_{1}$ and $F_{2}$ are fields. Then the following statements are equivalent. 
(1) $(\Omega(R))^{c}$ satisfies $K u_{2}$.

(2) $\left|F_{i}\right| \leq 5$ for $i=1,2$.

Proof. $\quad(1) \Rightarrow(2)$

As $(G(R))^{c}$ is a subgraph of $(\Omega(R))^{c}$, we obtain that $(G(R))^{c}$ satisfies $K u_{2}$. So, by Lemma 11, it follows that $\left|F_{i}\right| \leq 5$ for $i=1,2$.

\section{$(2) \Rightarrow(1)$}

Now $R=F_{1} \times F_{2}$, where $\left|F_{i}\right| \leq 5$ for $i=1,2$. Note that $\left\{M_{1}=(0) \times F_{2}, M_{2}=F_{1} \times(0)\right\}$ is the set of all maximal ideals of $R$ with $\left|M_{i}\right| \leq 5$ for $i=1,2$. Let $V_{1}=M_{1} \backslash\{(0,0)\}$, $V_{2}=M_{2} \backslash\{(0,0)\}, V_{3}=\{(0,0)\}$, and $V_{4}=F_{1} \backslash(0) \times F_{2} \backslash(0)$. Let $g_{i}(i=1,2)$ be the subgraph of $(\Omega(R))^{c}$ induced on $V_{i}$. Observe that $g_{i}$ is a complete graph on at most four vertices for $i=1,2$ and each vertex of $V_{4}$ is an isolated vertex, and $(0,0)$ is adjacent to each vertex of $V_{1} \cup V_{2}$. Note that the vertex set of $(\Omega(R))^{c}=V_{1} \cup V_{2} \cup V_{3} \cup V_{4}$ and $V_{i} \cap V_{j}=\emptyset$ for all distinct $i, j \in\{1,2,3,4\}$. It is clear from the above description of $(\Omega(R))^{c}$ that it satisfies $K u_{2}$.

Remark 34. Let $R$ be a ring with exactly two maximal ideals. If $(\Omega(R))^{c}$ satisfies $K u_{1}$, then we obtain from (2) $\Rightarrow$ (1) of Theorem 32 that $(\Omega(R))^{c}$ satisfies $K u_{2}$. We mention below an example of a ring $R$ with exactly two maximal ideals such that $(\Omega(R))^{c}$ satisfies $K u_{2}$ but it does not satisfy $K u_{1}$.

Let $R=F_{1} \times F_{2}$, where $F_{1}$ is a field with $\left|F_{1}\right|=5$ and $F_{2}$ is a field with $\left|F_{2}\right| \leq 5$. Note that $\left\{M_{1}=(0) \times F_{2}, M_{2}=F_{1} \times(0)\right\}$ is the set of all maximal ideals of $R$ with $\left|M_{1}\right| \leq 5$ and $\left|M_{2}\right|=5$. As the subgraph of $(\Omega(R))^{c}$ induced on $M_{2}$ is a clique on five vertices, it follows that $(\Omega(R))^{c}$ does not satisfy $K u_{1}$ but by Lemma 33, we obtain that $(\Omega(R))^{c}$ satisfies $K u_{2}$.

Theorem 35. Let $R$ be a ring with exactly two maximal ideals. Then the following statements are equivalent.

(1) $(\Omega(R))^{c}$ satisfies $K u_{2}$.

(2) $R$ is isomorphic to one of the following rings.

(a) $F_{1} \times F_{2}$, where $F_{1}$ and $F_{2}$ are fields with $\left|F_{i}\right| \leq 5$ for $i=1,2$.

(b) $F_{1} \times R_{2}$, where $F_{1}$ is a field with $\left|F_{1}\right|=2$ and $\left(R_{2}, M_{2}\right)$ is a local ring which is not a field and $\left|R_{2}\right|=4$.

Proof. $\quad(1) \Rightarrow(2)$

As $(G(R))^{c}$ is a subgraph of $(\Omega(R))^{c}$, it follows that $(G(R))^{c}$ satisfies $K u_{2}$. Hence from Lemma 10 we obtain that $R \approx R_{1} \times$ $R_{2}$ as rings, where $\left(R_{i}, M_{i}\right)$ is a quasilocal ring for $i=1,2$. If both $R_{1}$ and $R_{2}$ are fields, then Lemma 33 implies that $R$ is isomorphic to the ring given in (a). If $R_{2}$ is not a field, then from Lemma 28, we obtain that $R$ is isomorphic to the ring described in (b).

$(2) \Rightarrow(1)$

(2) $\Rightarrow$ (1) follows immediately from Theorem 32 and Lemma 33.

Theorem 36. Let $R$ be a ring with exactly three maximal ideals. Then the following statements are equivalent.

(1) $(\Omega(R))^{c}$ satisfies $K u_{1}$.
(2) $R \approx \mathbf{Z}_{2} \times \mathbf{Z}_{2} \times \mathbf{Z}_{2}$ as rings.

(3) $(\Omega(R))^{c}$ satisfies $K u_{2}$.

(4) $(\Omega(R))^{c}$ is planar.

Proof. (1) $\Rightarrow(2)$ and (3) $\Rightarrow$ (2).

As $(G(R))^{c}$ is a subgraph of $(\Omega(R))^{c},(1) \Rightarrow(2)$ and (3) $\Rightarrow$

(2) follow from Theorem 24.

(2) $\Rightarrow(4)$

$(2) \Rightarrow(4)$ follows immediately from Lemma 31.

$(4) \Rightarrow$ (1) and (4) $\Rightarrow$ (3).

Indeed it follows from Kuratowski's theorem [8, Theorem 5.9] that $(\Omega(R))^{c}$ satisfies both $K u_{1}^{*}$ and $K u_{2}^{*}$.

\section{Characterization of Quasilocal Rings $R$ Which Are Not Fields Such That $(\Omega(R))^{c}$ Is Planar}

Let $(R, M)$ be a quasilocal ring. If $M=(0)$, then $R$ is a field. In such a case, each vertex of $(\Omega(R))^{c}$ is an isolated vertex and hence it is clear that $(\Omega(R))^{c}$ is planar. So, we assume that $M \neq(0)$. The main result of this section is Theorem 37 which characterizes quasilocal rings $(R, M)$ which are not fields such that $(\Omega(R))^{c}$ is planar.

Theorem 37. Let $(R, M)$ be a quasilocal ring which is not a field. Then the following statements are equivalent.

(1) $(\Omega(R))^{c}$ satisfies $K u_{1}$ and $K u_{2}$.

(2) $(\Omega(R))^{c}$ satisfies $K u_{1}$.

(3) $|M| \leq 4,|R / M| \leq 4$, and $|R| \leq 16$.

(4) $(\Omega(R))^{c}$ is planar.

Proof. $\quad(1) \Rightarrow(2)$ This is clear.

(2) $\Rightarrow(3)$.

As $(\Omega(R))^{c}$ satisfies $K u_{1}$, it follows that $|M| \leq 4$. Now $|R / M| \leq 4$ follows from Lemma 6 . Hence $|R|=|M||R / M| \leq$ $4 \times 4=16$ :

(3) $\Rightarrow(4)$

Now, $|M| \leq 4,|R / M| \leq 4$, and $|R| \leq 16$. Note that $(\Omega(R))^{c}$ is a graph with vertex set $R=U(R) \cup N U(R)$. Each unit of $R$ is an isolated vertex of $(\Omega(R))^{c}$ and as $|N U(R)| \leq 4$, it is clear that $(\Omega(R))^{c}$ is planar:

(4) $\Rightarrow(1)$

It follows from Kuratowski's theorem [8, Theorem 5.9] that $(\Omega(R))^{c}$ satisfies both $K u_{1}^{*}$ and $K u_{2}^{*}$.

Lemma 38. Let $(R, M)$ be a quasilocal ring which is not a field. Then the following statements are equivalent.

(1) $(\Omega(R))^{c}$ satisfies $K u_{2}$.

(2) $|M| \leq 5,|R / M| \leq 5$, and $|R| \leq 25$.

Proof. $\quad(1) \Rightarrow(2)$

As $(\Omega(R))^{c}$ satisfies $K u_{2}$, it follows that $|M| \leq 5$. Now $|R / M| \leq 5$ follows from Lemma 6 . Hence $|R|=|M||R / M| \leq$ $5 \times 5=25$.

(2) $\Rightarrow$ (1) 
Now $R=U(R) \cup N U(R)$ and $|N U(R)|=|M| \leq 5$. As each unit of $R$ is an isolated vertex of $(\Omega(R))^{c}$ and any graph on at most five vertices satisfies $K u_{2}$, it follows that $(\Omega(R))^{c}$ satisfies $K u_{2}$.

Example 39. Let $R=\mathbf{Z} / 25 \mathbf{Z}$. Note that $R$ is a local ring with unique maximal ideal $M=5 \mathbf{Z} / 25 \mathbf{Z}=\{0+25 \mathbf{Z}, 5+25 \mathbf{Z}, 10+$ $25 \mathbf{Z}, 15+25 \mathrm{Z}, 20+25 \mathrm{Z}\}$. As $|M|=5$, it is clear that $(\Omega(R))^{c}$ does not satisfy $K u_{1}$. Moreover, from Lemma 38, it follows that $(\Omega(R))^{c}$ satisfies $K u_{2}$.

Remark 40. With the help of theorems proved by Corbas and Williams in [9], Belshoff and Chapman in [10] have listed up to isomorphism, the local rings of order $p^{2}, p^{3}$ and all the local rings of order 16. From the above list, we mention below up to isomorphism of rings, all local rings which are not fields of order 4, 8, 9, 25 and all local rings $(R, M)$ with $|R|=16$ and $|M|=4$.

(1) Local rings of order 4 which are not fields are $\mathbf{Z}_{4}$ and $\mathbf{Z}_{2}[X] /\left(X^{2}\right)$.

(2) Local rings of order 8 which are not fields are $\mathbf{F}_{2}[X] /\left(X^{3}\right), \mathbf{F}_{2}[X, Y] /(X, Y)^{2}, \mathbf{Z}_{4}[X] /\left(2 X, X^{2}\right)$, and $\mathbf{Z}_{4}[X] /\left(2 X, X^{2}-2\right)$.

(3) Local rings of order 9 which are not fields are $\mathbf{Z}_{9}$ and $\mathbf{Z}_{3}[X] /\left(X^{2}\right)$.

(4) Local rings $(R, M)$ with $|R|=16$ and $|M|=4$ are $\mathbf{F}_{4}[X] /\left(X^{2}\right)$ and $\mathbf{Z}_{4}[X] /\left(1+X+X^{2}\right)$.

\section{Conflict of Interests}

The authors declare that there is no conflict of interests regarding the publication of this paper.

\section{Acknowledgment}

The authors are very much thankful to the Academic Editors for their suggestions and support.

\section{References}

[1] P. K. Sharma and S. M. Bhatwadekar, "A note on graphical representation of rings," Journal of Algebra, vol. 176, no. 1, pp. 124-127, 1995.

[2] H. R. Maimani, M. Salimi, A. Sattari, and S. Yassemi, "Comaximal graph of commutative rings," Journal of Algebra, vol. 319, no. 4, pp. 1801-1808, 2008.

[3] M. I. Jinnah and S. C. Mathew, "When is the comaximal graph split?" Communications in Algebra, vol. 40, no. 7, pp. 2400-2404, 2012.

[4] S. M. Moconja and Z. Z. Petrović, "On the structure of comaximal graphs of commutative rings with identity," Bulletin of the Australian Mathematical Society, vol. 83, no. 1, pp. 11-21, 2011.

[5] M. Ye and T. Wu, "Co-maximal ideal graphs of commutative rings," Journal of Algebra and Its Applications, vol. 11, no. 6, Article ID 1250114, 14 pages, 2012.
[6] A. Gaur and A. Sharma, "Maximal graph of a commutative ring," International Journal of Algebra, vol. 7, no. 9-12, pp. 581588, 2013.

[7] R. Balakrishnan and K. Ranganathan, A Textbook of Graph Theory, Springer, New York, NY, USA, 2000.

[8] N. Deo, Graph Theory with Applications to Engineering and Computer Science, Prentice Hall of India Private Limited, New Delhi, India, 1994.

[9] B. Corbas and G. D. Williams, "Rings of order $p^{5}$. I. Nonlocal rings," Journal of Algebra, vol. 231, no. 2, pp. 677-690, 2000.

[10] R. Belshoff and J. Chapman, "Planar zero-divisor graphs," Journal of Algebra, vol. 316, no. 1, pp. 471-480, 2007. 


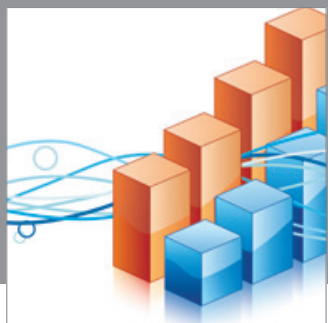

Advances in

Operations Research

mansans

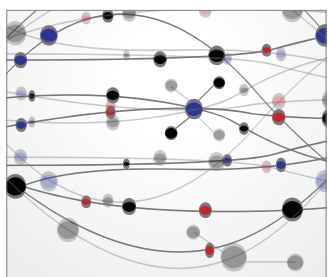

The Scientific World Journal
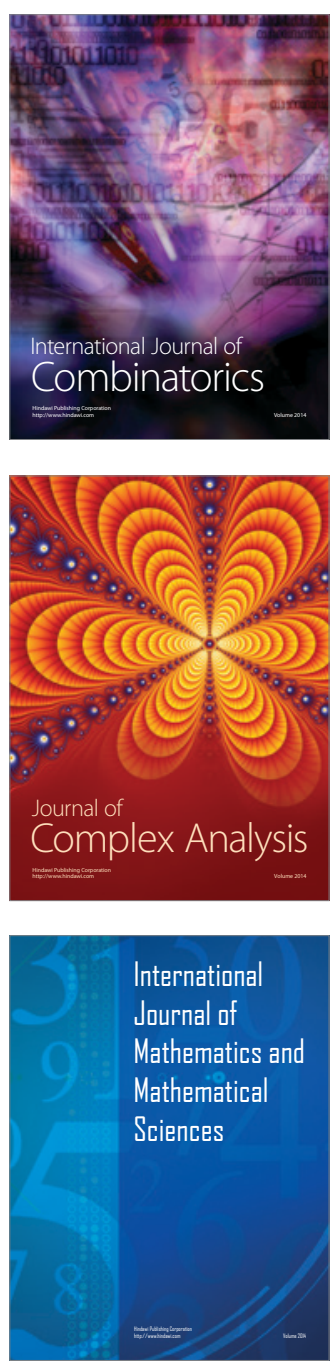
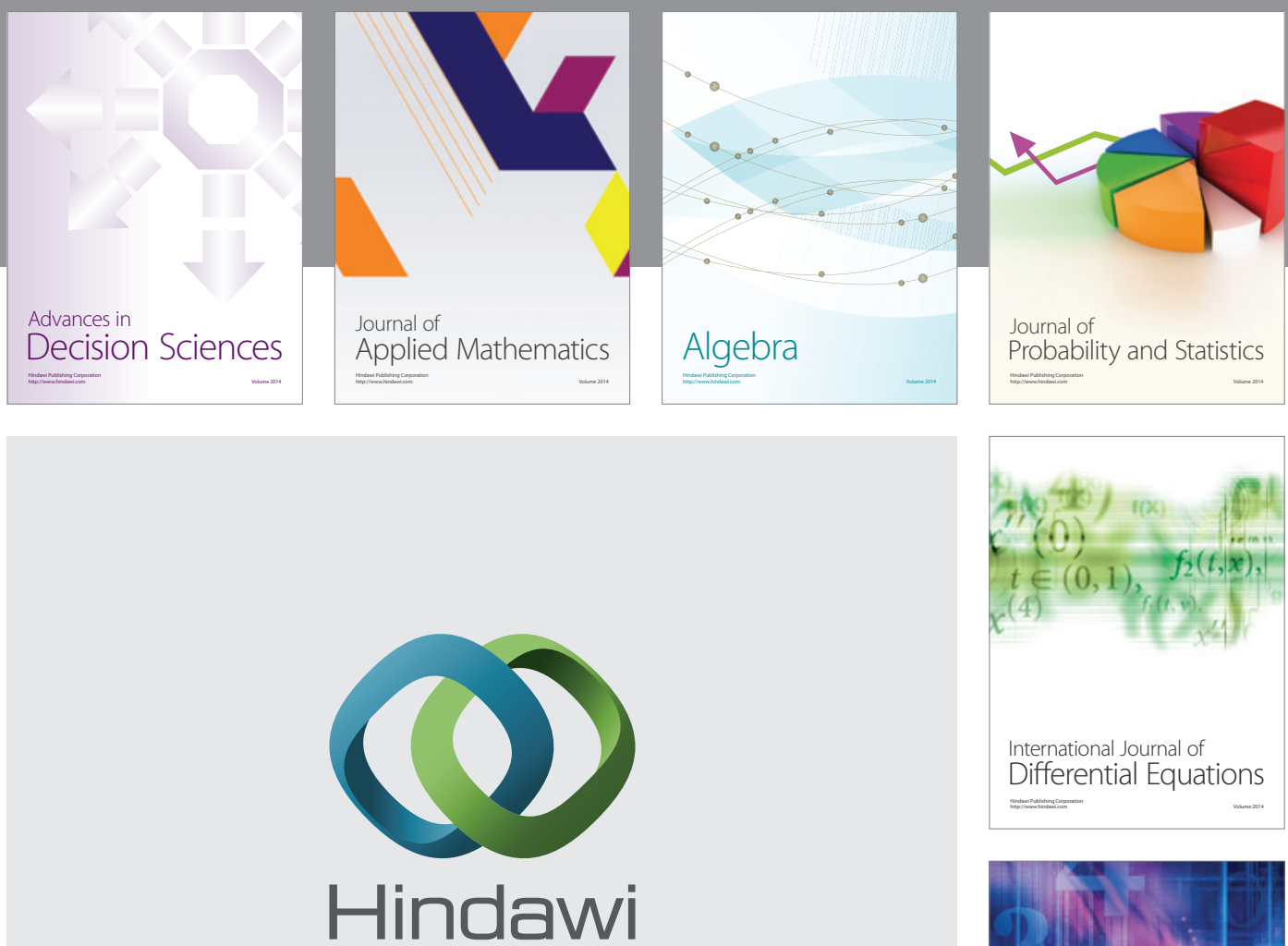

Submit your manuscripts at http://www.hindawi.com
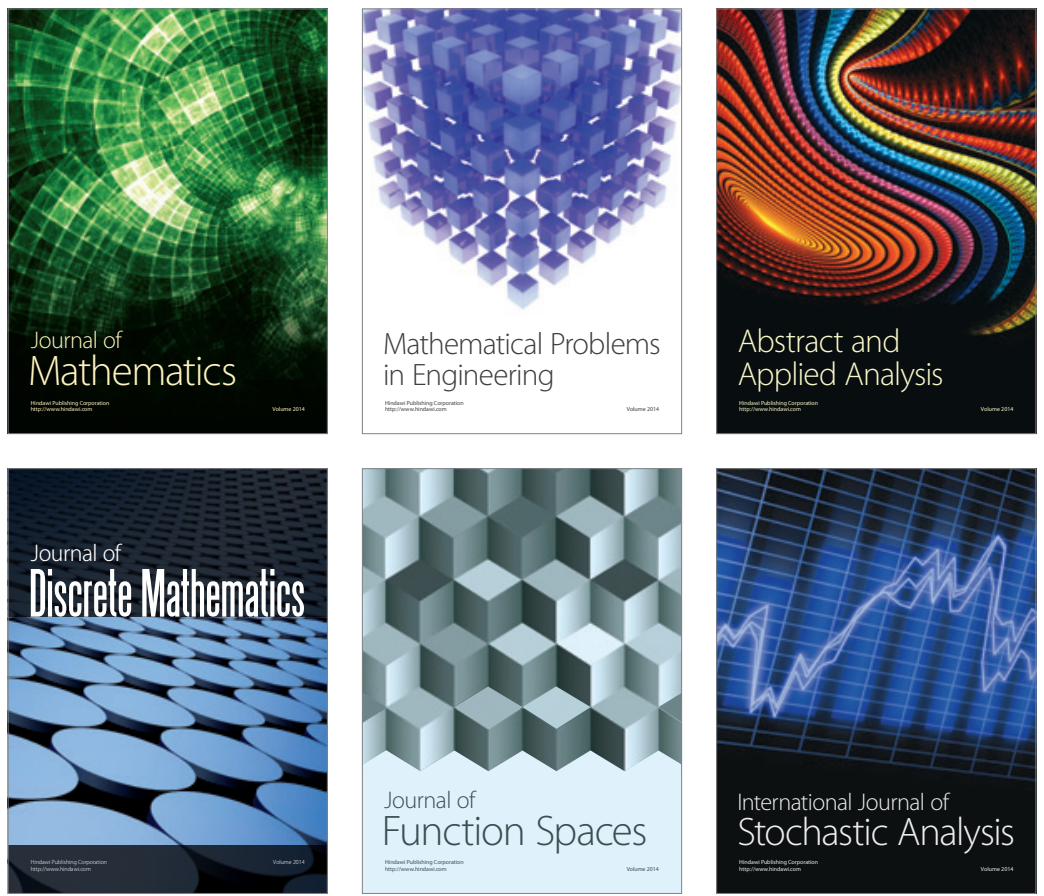

Journal of

Function Spaces

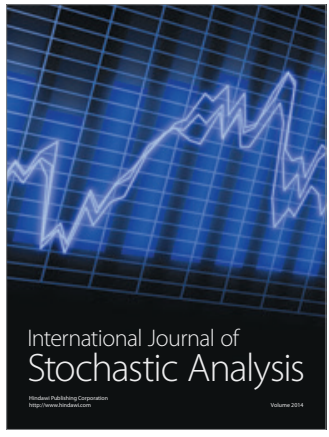

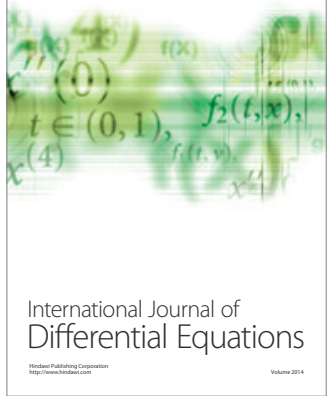
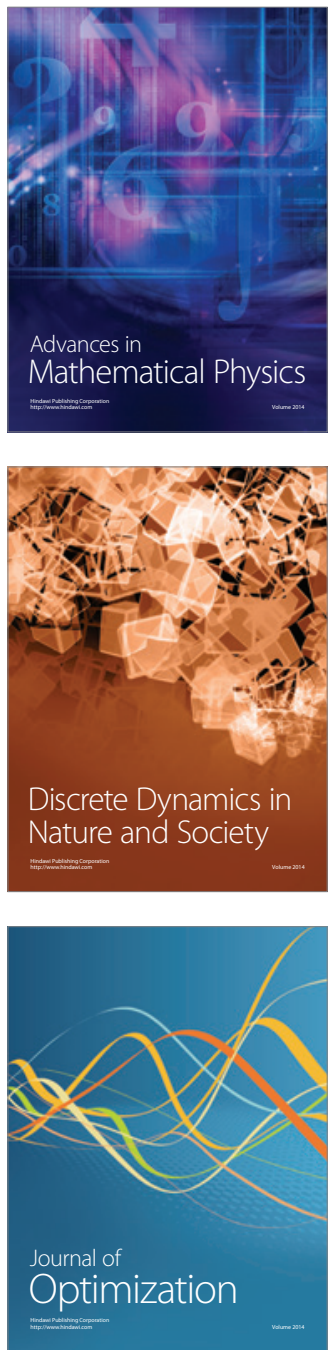